\title{
Future Directions of Regional Anaesthesia
}

\author{
André Van Zundert ${ }^{1}$, Sandeep Diwan ${ }^{2}$ \\ ${ }^{1}$ Department of Anaesthesiology, The University of Queensland, Brisbane, Australia. \\ ${ }^{2}$ Department of Anaesthesiology, Sancheti Hospital, Pune, Maharashtra, India.
}

Since the $19^{\text {th }}$ century, we have seen general and regional anaesthesia develop as complementary fields rather than opponents to provide high-quality anaesthesia and analgesia for patients. Combination of the two techniques often results in better outcomes with decreased incidence of adverse effects. General and regional anaesthesia, or loco-regional anaesthesia alone provide patient benefits including a pain free recovery.

Anaesthesiologists would want to be acquainted with regional techniques that would benefit the patient and cause the least harm. In fact, the key question is what the best solution is for a particular patient considering current problems, the type of surgical intervention, the available skills and support from the surgeon and anaesthetist. Many surgeons are unaware of the possibility of combining regional and general anaesthesia. Also, local practice differs in different parts in the world. In some countries, patients are well-informed and know what to expect during regional anaesthesia blocks and stay awake during the whole procedure (regional block and surgical intervention), or appreciate some distraction using a headphone with their favourite music. In other countries, patients want to receive sedation or even general anaesthesia during the regional anaesthesia procedure and/or during the surgical intervention as they prefer to be unaware of the whole procedure.

Nevertheless, patients expect to get superb service from a skilled and experienced anaesthetist in a wide range of regional anaesthesia techniques demonstrating extensive knowledge in applied anatomy, pharmacology, toxicology, monitoring, and expect no less than a perfect pain-free technique without complications and a quick recovery. However, regional anaesthesia is not always perfect. In general anaesthesia, any deviation from the normal can easily be managed in the unconscious patient. In awake patients undergoing regional anaesthesia, complications of regional blockade may be recognized by the patient, which could be stressful for the patient.

In learning regional anaesthesia techniques, there is no substitute for personal tuition while performing numerous interventions from experienced practitioners. However, trainees need to study textbooks and attend workshops and conferences on regional anaesthesia to perfect techniques, as well as accessing online courses, seminars, video clips, apps, guidelines from professional societies (e.g. AORA) and journal articles.

Ultrasound-guided techniques are preferred and superior to blind techniques, allowing more precise localisation of nerves (peripheral nerve blocks) or location of the subarachnoid and epidural space (central neuraxial anaesthesia). The International Journal of Regional Anaesthesia (IJRA), an official publication of AORA is such a specialised journal offering peer-reviewed articles on a variety of topics focussed on local-regional anaesthesia and pain with an emphasis on visualisation of the technique using colourful imaging to illustrate anatomical and other practical aspects. As the scope of the IJRA expands, the Editorial Board aims to bring up-to date reliable and practical information for the practitioner with clinical articles, review articles, Letters-to-the Editor, but also on updates of books, e-books, atlases, apps, videos, infograms and provide website links to guidelines, useful for the practising on regional anaesthesia.

Newer block techniques have evolved over time including use of ultrasound techniques. Anaesthetists need to master a variety of regional blocks including central neuraxial and peripheral nerve blocks to be qualified in regional anaesthesia.

Regional anaesthesia plays an essential role in our practice. Some golden rules apply to maximise safety and efficacy: a) Discuss the regional blockade with the patient, explaining benefits and risks and obtain informed written consent; b) Discuss with the surgeon what procedure you intend to perform and the site of incision; c) Discuss with the patient any potential complication/side effect and document these in your anaesthesia chart; d) Perform the regional block with the best intention for

Address of Correspondence: Prof. André Van Zundert, Professor \& Chairman Discipline of Anaesthesiology, The University of Queensland, Brisbane, Australia. E-mail:vanzundertandre@gmail.com

DOI: 10.13107/ijra.2021.v02i01.020 | (C) 2021 International Journal of Regional Anaesthesia | Available on www.ijrajournal.com | This is an Open Access article distributed under the terms of the Creative Commons Attribution Non-Commercial License (http://creativecommons.org/licenses/by-nc/3.0) which permits unrestricted non-commercial use, distribution, and reproduction in any medium, provided the original work is properly cited.

How to cite this article: Van Zundert A, Diwan S | Future Directions of Regional Anaesthesia | International Journal of Regional Anaesthesia | January-June 2021; 2(1): 17-18. 
the patient, not for the best interest of the anaesthetist; e) Perform regional anaesthesia blocks in an appropriate setting (wellequipped, adequately-staffed, safe environment) capable of handling complications (ventilator at hand; resuscitation drugs/equipment/Intralipid at hand) with intravenous access in situ, applying adequately-monitored according standards; f) Always fractionate any doses, check their impact on the patient, and respect dose limits; g) Document the surgical intervention, positioning of the patient on the operating table and record any problem/complication (e.g., haemorrhage, pneumothorax and paraesthesia); h) In case of a neurological complication check the patient yourself and refer to a neurologist at an early stage; and i) Always have a plan B, in case of an unsuccessful block. Infrastructure and ergonomics play an important part in success of the block. Similarly, important is to avoid wrong-route, wrongdose, wrong-side, wrong-site injections and to carefully label all connections and tubing. Management of paediatric patients requires even greater efforts as children cannot be relied upon to ask for analgesia during a procedure. Regional anaesthesia techniques are excellent tools also in children, but these procedures themselves can be painful. In obstetric anaesthesia, pain relief during childbirth may be stressful with the woman in full labour requiring an immediate epidural.

The growing popularity of ultrasonography is a very welcome addition to regional anaesthesia and allows more precision application in regional anaesthesia, in particular peripheral nerve blocks. It provides bedside imaging and dynamic assessment for nerve localisation and target-specific injections, visualising needle advancement in real time and observation of local anaesthetic spread around nerve structures. Use of ultrasound is an evolving aspect of our specialty, offering major advantages and superiority over blind techniques, such as real-time visualisation of soft tissues, muscles, nerves, veins and arteries, improving safe practice. Shortcomings with ultrasound include limited resolution at deep levels especially in the obese, and artefacts created by bone structures. Anaesthetists practising regional anaesthesia with ultrasound need to know the basic principles of ultrasound imaging and knobology, regional anatomy specifically related to interventional procedures, ultrasound scanning and image interpretation and the technical considerations for needle insertion and injection (step-by-step, easy-to-follow, how-to-do-it instructions). Whether ultrasonography can be further improved in obtaining the best possible resolution of the area, ruling out the need for extra monitoring devices (e.g., nerve stimulator) and landmark techniques is still under debate. It is wise however, to limit the injection pressure to 15 psi using an injection pressure monitor device.

NYSORA (New York Society of Regional Anaesthesia) recently introduced the Next Level CMETM programme (https://nextlevelcme.com). This educational and technology entity provides a personalised and boutique learning experience for medical practitioners utilising a custom-built eLearning platform and a range of propriety cognitive aids, illustrations and animations. The aim is to have all your study materials organised in one place, accessible anytime anywhere. NexLevel CMETM allows the practitioner to create their own customised and condensed study scripts (personal, departmental, region, country) in minutes to make learning faster and more engaging. The focus is on an in-depth, customised complete training portfolio in anaesthesia, for point of care ultrasound and pain and perioperative medicine, which allows control of learning processes.

IJRA is a distinguished journal, carefully prepared by the Editorial team of dedicated anaesthetists interested in regional anaesthesia, for those medical practitioners who want to learn extra steps in regional anaesthesia practice for them and patients' benefit.

We hope you'll find in this issue of IJRA comprehensive, inspiring and practical information about regional anaesthesia and nerve block techniques with clinical applicability that will influence the professional lives of many colleagues. It is important to remember that injection of the local anaesthetic drugs is just the beginning and not the end of the anaesthetic. When this approach is followed considerable benefits can accrue to the patient.

\section{Aspecial thanks goes to..... \\ AORA}

Chairman: Dr. TVS Gopal, Dr. J Balvenkatasubramanian

President: Dr. Vrushali Ponde;

Vice President: Dr. Sudhakar Koppad;

Core Committee: Dr. Satish Kulkarni, Dr. Ashit Mehta, Dr.

Javed Khan;

Executive Committee.

IJRA

Editorial Team, Editorial Committee Members, International Executive Committee Members, Author and Contributor to the Articles.

Dr. Ashok Shyam.

Journal Coordinator \& Academic Research Group (Journal Publisher) 